\title{
Channel Calibration based on Correlation Analysis for Multichannel SAR-GMTI Systems with Performance Test
}

\author{
Zhao-Yan Chen ${ }^{\mathrm{a}}$, Tong Wang, ${ }^{\mathrm{b}, *}$ \\ ${ }^{a}$ School of Computer Science and Technology, Shanghai Business School,Shanghai 201400,People's Republic of China \\ ${ }^{b}$ National Laboratory of Radar Signal Processing,Xidian University, Xi'an 710071, People's Republic of China
}

\begin{abstract}
This paper proposes an accurate channel calibration algorithm for ground moving target indication (GMTI) applications with multichannel synthetic aperture radar (SAR) systems. The proposed algorithm is developed in a statistical fashion, and a useful tool called correlation analysis is employed, which measures the coherence between two channels by means of correlation coefficient. Based on correlation analysis theory, an optimum solution to a very general channel calibration problem is obtained, for which only the knowledge of the first two moments of the channel output signals is assumed and no other distributional assumptions are made. The proposed optimum solution represents a generic solution to the general channel calibration problem, which leads to a simple implementation form and needs no iterative operation. The performance test results show that the proposed correlation analysis processing algorithm is an effective way of calibrating the channels.
\end{abstract}

Keywords: channel calibration; correlation analysis; Along-Track Interferometry (ATI); Ground Moving Target Indication (GMTI); Synthetic Aperture Radar (SAR)

(Submitted on October 29, 2017; Revised on November 12, 2017; Accepted on December 1, 2017)

(C) 2017 Totem Publisher, Inc. All rights reserved.

\section{Introduction}

Multichannel synthetic aperture radar (SAR) is a viable means of performing ground moving target indication (GMTI). In recent years, SAR-GMTI has stirred a great deal of interest [5,10,14,15]. With several channels or antennae aligning along the platform track, along-track interferometry (ATI) technique [13] can be used to accomplish the GMTI function. For a multichannel SAR-GMTI system, it is desirable to have a high coherence between the channels to obtain a good GMTI performance. However, in practical applications, the decorrelation of the channel output signals is inevitable, which can lead to the coherence loss and therewith the GMTI performance loss.

Several decorrelation sources are summarized as follows:

1) Thermal noise. Since the thermal noise components in each channel are statistically independent, the existence of noise will decrease the channel coherence. However, one can alleviate this kind of decorrelation effect by increasing the clutter-to-noise ratio (CNR).

2) Clutter internal motion. In vegetated areas, the clutter internal motion is inevitable, and this will result in a loss of coherence.

3) Channel mismatch. The channels may have different system transfer functions. This kind of decorrelation is mainly caused by the non-ideal hardware characterization. Furthermore, some uncontrollable natural factors such as temperature can also introduce additional decorrelation.

4) Antenna pattern differences. In practice, antenna beam patterns may be different from channel to channel due to the imperfections. In sidelobe regions, the channel data will be uncorrelated due to the low CNR. Moreover, antenna deformation and installation errors may introduce beam mispointing errors, and these will aggravate the decorrelation. 
5) Phase center offset. In practice, if the displaced phase center antenna (DPCA) condition [16] is not met, the existed phase center offset will introduce a DPCA lag time, and this will decrease the channel coherence. However, this kind of decorrelation can be compensated when the effective ATI-baseline is known.

6) Phase center location uncertainties. For airborne applications, both the intrinsic uncertainties of the radar platform heading and the changing weather conditions can result in yaw or pitch errors, which will lead to a misalignment of the antenna axis with the platform velocity. These kinds of uncertainties will introduce random interferometric phase errors and therewith the decorrelation.

7) Moving targets. If moving targets are presented, the target and the clutter signatures will compete against one another, and this will introduce a coherence loss. However, this kind of decorrelation feature should be preserved for GMTI purpose.

The decorrelation sources listed above can lead to inevitable channel errors, which can be viewed as random signals. These random errors are summarized as channel imbalances. Calibrated channel data are essential for clutter suppression methods such as ATI, DPCA [12] and space-time adaptive processing (STAP) [7], although the systematic phase term caused by the baseline should be preserved first for the STAP method [9]. As such, the channel calibration problem must be addressed before the GMTI processing. A popular channel calibration algorithm called adaptive two-dimensional calibration (A2DC) was originally presented in [7], which iteratively calibrates the channels in two-dimensional (2-D) frequency domain. Furthermore, a simple method named de-trending is presented in [9] to remove the interferometric phase ramp, but the amplitude imbalance still remains uncompensated. Thus, the A2DC method is preferred when the compensation of amplitude imbalance is crucial.

The above mentioned methods are performed in a single processing domain. There is therefore the limitation that the channel errors can only be compensated in a single processing domain, and if there are errors that cannot be compensated in this single processing domain, these methods will become inefficient. As such, a two-stage channel calibration technique is proposed in [1] to calibrate the channels in a more complicated case, where the channel errors can be classified into two categories, that is, the so called convolutional and multiplicative errors. In [1], the convolutional errors are compensated by a Stage 1 calibration filter, in which the conventional 2-D frequency domain calibration methods can be employed, and the multiplicative errors are compensated by a Stage 2 filter.

In this paper, we propose an accurate channel calibration algorithm, which can serve as an alternative for the Stage 1 calibration filter presented in [1]. However, for the sake of conciseness, we only focus on a simple calibration case in which a single domain is processed with the A2DC or the proposed method. In this paper, a very general channel calibration problem is considered first, for which only the knowledge of the first two moments of the channel output signals is assumed and no other distributional assumptions are made. Then the algorithm is developed in a statistical fashion. It should be pointed out that, there are two kinds of statistical analysis methods. The first one is the regression analysis, in which only one variable is considered random. The second one is the correlation analysis, in which two or even more variables are considered random. It is important to note the distinction between these two kinds of statistical analysis methods, which can lend much insight to the proposed method. Based on correlation analysis theory, an optimum solution to the general channel calibration problem is presented, which leads to a simple and accurate channel calibration algorithm. Furthermore, the practical implementation issue of the proposed method is also addressed. The real raw data from an experimental SARGMTI system are used to test the performance of the proposed method. First, the channel calibration results of the proposed method are provided. Some quality measures are employed to test the performance. Second, the comparative results are illustrated to show the performance improvement. Results with real SAR data show that the proposed method is an effective way of calibrating the channels.

This paper is organized as follows. In Section 2, the concepts of channel coherence, clutter suppression ratio (CSR), and channel imbalances are introduced, which give rise to the need for the proposed algorithm. In Section 3, the proposed algorithm is introduced to solve the general channel calibration problem, and the practical implementation issue is also discussed. In Section 4, the performance of the proposed algorithm is tested with real SAR data. In Section 5, some conclusions are drawn.

\section{Problem formulation}

In this section, the concept of channel coherence is introduced, and its impact on the clutter suppression performance is highlighted, which give rise to the channel calibration problem. For the sake of signal-to-noise-ratio-optimal (SNR-optimal) coherent processing, in the following analysis, it is assumed that the channel output signals are already range compressed and range cell migration corrected. 


\subsection{Coherence and its impact on clutter suppression performance}

From a channel-calibration point of view, it is convenient to have a discussion involving only two channels. Without loss of generality, we denote the output 2-D time domain signals of channels 1 and 2 as $z_{1}\left(t_{\tau}, t_{\eta}\right)$ and $z_{2}\left(t_{\tau}, t_{\eta}\right)$, respectively, with $t_{\tau}$ denotating range time and $t_{\eta}$ azimuth time. As such, let $z_{1}\left(t_{\tau}, t_{\eta}\right)$ and $z_{2}\left(t_{\tau}, t_{\eta}\right)$ be two zero-mean jointly distributed 2-D random signals with auto-power spectral densities (auto-PSDs) $p_{11}\left(f_{\tau}, f_{\eta}\right)$ and $p_{22}\left(f_{\tau}, f_{\eta}\right)$ and with cross-PSD $p_{12}\left(f_{\tau}, f_{\eta}\right)$, where $f_{\tau}$ and $f_{\eta}$ denote range and Doppler frequencies, respectively.

By taking 2-D fast Fourier transform (FFT) of $z_{1}\left(t_{\tau}, t_{\eta}\right)$ and $z_{2}\left(t_{\tau}, t_{\eta}\right)$, the 2-D frequency domain signals $Z_{1}\left(f_{\tau}, f_{\eta}\right)$ and $\mathrm{Z}_{2}\left(\mathrm{f}_{\tau}, \mathrm{f}_{\eta}\right)$ are obtained. The normalized form of cross-PSD is the complex valued correlation coefficient in frequency domain, which is given by [21]:

$$
\gamma_{12}\left(f_{\tau}, f_{\eta}\right)=\frac{p_{12}\left(f_{\tau}, f_{\eta}\right)}{\sqrt{p_{11}\left(f_{\tau}, f_{\eta}\right) p_{22}\left(f_{\tau}, f_{\eta}\right)}}=D_{12}\left(f_{\tau}, f_{\eta}\right) \exp \left\{j \phi_{12}\left(f_{\tau}, f_{\eta}\right)\right\}
$$

where $D_{12}\left(f_{\tau}, f_{\eta}\right)$ is the degree of coherence (DOC) [17] or the coherence magnitude in the 2-D frequency domain, which represents the amount of linear coherency between $Z_{1}\left(f_{\tau}, f_{\eta}\right)$ and $Z_{2}\left(f_{\tau}, f_{\eta}\right)$, and it can be given by

$$
D_{12}\left(f_{\tau}, f_{\eta}\right)=\frac{\left|p_{12}\left(f_{\tau}, f_{\eta}\right)\right|}{\sqrt{p_{11}\left(f_{\tau}, f_{\eta}\right) p_{22}\left(f_{\tau}, f_{\eta}\right)}}
$$

and the argument $\phi_{12}\left(f_{\tau}, f_{\eta}\right)$ is the phase lag or lead of this coherency. In fact, $\phi_{12}$ is the interferometric phase ramp in the Doppler frequency domain, which is caused by the effective ATI-baseline, and this phase ramp should be removed via channel calibration processing for DPCA based clutter suppression.

The DOC measures the similarity between the two signals $z_{1}$ and $z_{2}$. In SAR-GMTI applications, this kind of similarity mainly comes from the clutter component due to the intrinsic strong coherency of the stationary clutter, and only a little similarity comes from the moving targets owing to the decorrelation effect of the target motion, whereas none from the uncorrelated noises. Therefore, the DOC can be employed as a metric to evaluate the clutter suppression performance. More specifically, if $\mathrm{w}_{\mathrm{opt}}$ is the optimum filter that minimizes the mean square error (MSE):

$$
E\left[|e|^{2}\right]=E\left[\left|z_{1}-w z_{2}\right|^{2}\right]
$$

where $E[\cdot]$ denotes the mathematical expectation operator, and $|x|$ represents the absolute value of $x$, then the cluttersuppressed output signal $e$ can be written as

$$
e=z_{1}-w_{o p t} z_{2}
$$

and the auto-PSD of $e$ is given by [8]:

$$
p_{e e}\left(f_{\tau}, f_{\eta}\right)=\left[1-D_{12}^{2}\left(f_{\tau}, f_{\eta}\right)\right] p_{11}\left(f_{\tau}, f_{\eta}\right)
$$

For an intuitive interpretation of (5), note that when the DOC is very high, the clutter component of $z_{1}$ is very "similar" to the one of $z_{2}$, thus the clutter can be suppressed very well, and therefore the residual clutter power is low. On the contrary, when the DOC is very low, the similarity between the clutter components of $z_{1}$ and $z_{2}$ is also very low, thus $z_{2}$ makes little contribution to the suppression of the clutter component of $z_{1}$.

The clutter suppression performance can be examined by the CSR, which is defined as the ratio of pre- to postsuppression clutter power. The analytical expression of the CSR in $\mathrm{dB}$ can be expressed as [20]:

$$
C S R=10 * \log 10\left(\frac{1}{1-D_{12}^{2}}\right)
$$


where $\log 10(\cdot)$ denotes the common base $10 \operatorname{logarithm}$, and $D_{12}$ is the DOC of the two channels. Note that we have here dropped the 2-D index which was used in the previous analysis. The reason is that the channel coherence is not affected by linear operations [8], thus $D_{12}$ remains the same in both 2-D time and 2-D frequency domains. As pointed out in [20], the $C S R$ is only $7.21 \mathrm{~dB}$ when $D_{12}=0.9$, and $17.01 \mathrm{~dB}$ when $D_{12}=0.99$. Therefore, the channel coherence should be improved to a certain level that is high enough to suppress the clutter sufficiently, and this gives rise to the channel calibration problem.

\subsection{Channel imbalances description}

It has been pointed out previously that the errors introduced by the decorrelation sources can be viewed as random signals and cannot be known a priori. As such, we lump together all these error terms into a channel imbalance term to carry out our analysis, and this is interpreted in detail in the following.

The 2-D frequency domain signals $Z_{1}\left(f_{\tau}, f_{\eta}\right)$ and $Z_{2}\left(f_{\tau}, f_{\eta}\right)$ can be approximately related as

$$
Z_{1}\left(f_{\tau}, f_{\eta}\right) \approx \alpha\left(f_{\tau}, f_{\eta}\right) Z_{2}\left(f_{\tau}, f_{\eta}\right)
$$

where $\alpha\left(f_{\tau}, f_{\eta}\right)$ denotes the channel imbalances between the two channels, and it can be expressed as

$$
\alpha\left(f_{\tau}, f_{\eta}\right)=\left|\alpha\left(f_{\tau}, f_{\eta}\right)\right| \exp \left\{j \phi_{12}\left(f_{\tau}, f_{\eta}\right)\right\}
$$

with $\left|\alpha\left(f_{\tau}, f_{\eta}\right)\right|$ denoting the amplitude spectrum imbalance, and $\phi_{12}\left(f_{\tau}, f_{\eta}\right)$ denoting the phase spectrum imbalance, which is in fact the phase ramp shown in (1).

The approximation is made in (7) because the relationship only holds in a statistical fashion when a considerable corruption is considered, and the corruption is introduced by the random errors from various decorrelation sources. Thus the channel imbalances are considered as stochastic processes. For SAR-GMTI applications, both amplitude- and phasespectrum imbalances should be removed by channel calibration processing. In the next section, we will develop a simple and accurate channel calibration algorithm to compensate these imbalances.

\section{Proposed channel calibration algorithm}

In this section, we propose a simple and accurate algorithm based on correlation analysis theory to resolve the channel calibration problem posed earlier. The proposed algorithm represents an optimum solution to the general channel calibration problem, which both leads to simple implementation procedures and requires the knowledge of the first two moments only. Finally, we discuss the practical implementation issue of the proposed method.

\subsection{Optimum solution to the general channel calibration problem}

We are aiming at designing an algorithm for dealing with both the amplitude and phase spectrum imbalances. As described previously, the measured signals are corrupted by unavoidable channel errors or imbalances coming from various sources. In a general channel calibration problem, the task is to calibrate the channels with the measured signals only, without knowing the imbalances. To put the problem in more practical terms, we turn our attention to a fairly general statistical model in solving the channel calibration problem: only the knowledge of the first two moments of the random signals is assumed, and no other distributional assumptions are made. Therefore, it is desirable to develop a channel calibration algorithm that both leads to simple implementation procedures and requires very little knowledge of only the first two moments. As such, we consider the theory of correlation analysis, which attempts to measure the strength of the relationship between two random signals by means of correlation coefficient [19]. Based on correlation analysis, the so called correlation analysis processing (CAP) algorithm is proposed to calibrate the channels. Details of the proposed algorithm are given in the following.

To begin, let channel 1 be the reference or master channel, and channel 2 be the slave channel that should be adjusted to the channel 1. For notational expediency, we drop the 2-D index $\left(\mathrm{f}_{\tau}, \mathrm{f}_{\eta}\right)$ that has been used in the previous analysis. The channel calibration processing can be described as

$$
Z_{2, c a l}=\beta Z_{2}
$$


where $Z_{2, \text { cal }}$ is the calibrated signal, and $Z_{2}$ is the signal to be calibrated or adjusted, and $\beta$ should be a coefficient that can calibrate the two channels, i.e., $\beta$ should satisfy the following relation

$$
Z_{1} \approx Z_{2, \text { cal }}=\beta Z_{2}
$$

where $Z_{1}$ is usually employed as a training signal or reference signal to construct the calibration coefficient $\beta$. The approximation is made in (10) because the relationship only holds in a statistical fashion. Because both $Z_{1}$ and $Z_{2}$ are considered random, the problem presented in (10) is a classic correlation analysis problem. As such, we should choose a coefficient $\beta$ so that $Z_{2, \text { cal }}$ has the largest possible common clutter signatures with $Z_{1}$, and in this way, $Z_{1}-Z_{2, \text { cal }}$ will only contain the signatures that are uncorrelated with $Z_{2}$. From a statistical point of view, that is to say, we want to choose a coefficient $\beta$ so that $Z_{1}-Z_{2, c a l}$ is uncorrelated with $Z_{2}$, and this can be expressed as

$$
E\left[\left(Z_{1}-Z_{2, \text { cal }}\right) Z_{2}^{*}\right]=0
$$

Inserting (9) into (11) and after some simple algebra yields

$$
\beta=\frac{E\left[Z_{1} Z_{2}^{*}\right]}{E\left[\left|Z_{2}\right|^{2}\right]}
$$

and this is the coefficient defined by the proposed CAP algorithm, which has a very simple form. After channel calibration with the proposed algorithm, the calibrated signal in (9) is given by

$$
Z_{2, c a l}=\frac{E\left[Z_{1} Z_{2}^{*}\right]}{E\left[\left|Z_{2}\right|^{2}\right]} Z_{2}
$$

So far, we have developed an optimum solution for the general channel calibration problem. From a practical point of view and insofar as the computation of the coefficient $\beta$ is concerned, it is meaningful to highlight the physical mechanism of the proposed algorithm. For clarity, the notation $\left(f_{\tau}, f_{\eta}\right)$ is retrieved. A physical interpretation of the optimum solution presented in (12) can be given using the definitions of the cross-PSD in 2-D frequency domain [18]:

$$
p_{12}\left(f_{\tau}, f_{\eta}\right) \triangleq E\left[Z_{1}\left(f_{\tau}, f_{\eta}\right) Z_{2}^{*}\left(f_{\tau}, f_{\eta}\right)\right]
$$

and the auto-PSD [18]:

$$
p_{22}\left(f_{\tau}, f_{\eta}\right) \triangleq E\left[Z_{2}\left(f_{\tau}, f_{\eta}\right) Z_{2}^{*}\left(f_{\tau}, f_{\eta}\right)\right]=E\left[\left|Z_{2}\left(f_{\tau}, f_{\eta}\right)\right|^{2}\right]
$$

Using (1), (14) and (15), one can rewrite (12) as

$$
\beta\left(f_{\tau}, f_{\eta}\right)=\frac{p_{12}\left(f_{\tau}, f_{\eta}\right)}{p_{22}\left(f_{\tau}, f_{\eta}\right)}=\left|\beta\left(f_{\tau}, f_{\eta}\right)\right| \exp \left\{j \phi_{12}\left(f_{\tau}, f_{\eta}\right)\right\}
$$

where $\left|\beta\left(f_{\tau}, f_{\eta}\right)\right|$ is the compensated amplitude spectrum imbalance and is given by

$$
\left|\beta\left(f_{\tau}, f_{\eta}\right)\right|=\frac{\left|p_{12}\left(f_{\tau}, f_{\eta}\right)\right|}{p_{22}\left(f_{\tau}, f_{\eta}\right)}=D_{12}\left(f_{\tau}, f_{\eta}\right) \sqrt{\frac{p_{11}\left(f_{\tau}, f_{\eta}\right)}{p_{22}\left(f_{\tau}, f_{\eta}\right)}}
$$

and $\phi_{12}\left(f_{\tau}, f_{\eta}\right)$ is the compensated phase spectrum imbalance which is the phase ramp shown in (1).

Finally, we highlight two particular properties of the proposed algorithm. In Appendix, we show that the proposed algorithm is optimum in both minimum mean square error (MMSE) [11] and best linear unbiased estimator (BLUE) [11] senses. The first property means that the proposed algorithm can calibrate the channels in the sense of minimizing the MSE, and the second property means that the proposed algorithm can obtain a linear, unbiased, and minimum variance estimate of the reference channel. 


\subsection{Practical implementation issue}

To put the proposed algorithm in more practical terms, it is necessary to estimate the values of the different expectation operations involved in (12) by means of the ensemble average. Unfortunately, in many practical SAR-GMTI applications only a single pass is employed, and thus only one realization of the two random signals $Z_{1}$ and $Z_{2}$ is available, whereas a large number of realizations are required to obtain a consistent estimate of each ensemble average involved in (12). However, under the assumption of ergodicity, it is reasonable to substitute the ensemble averages with the sample averages, which are obtained over a limited area surrounding the bin of interest. Hence it is possible to implement the proposed algorithm over a 2-D moving-window with dimension $n_{\tau} \times n_{\eta}$ in the 2-D frequency plane. As such, the coefficient in (12) can be estimated by

$$
\hat{\beta}\left(k_{\tau}, k_{\eta}\right)=\frac{\sum_{m_{\tau}=-\left(n_{\tau}-1\right) / 2}^{\left(n_{\tau}-1\right) / 2} \Sigma_{m_{\eta}=-\left(n_{\eta}-1\right) / 2}^{\left(n_{\eta}-1\right) / 2} Z_{1}\left(k_{\tau}-m_{\tau} \Delta_{\tau}, k_{\eta}-m_{\eta} \Delta_{\eta}\right) Z_{2}^{*}\left(k_{\tau}-m_{\tau} \Delta_{\tau}, k_{\eta}-m_{\eta} \Delta_{\eta}\right)}{\sum_{m_{\tau}=-\left(n_{\tau}-1\right) / 2}^{\left(n_{\tau}-1\right) / 2} \Sigma_{m_{\eta}=-\left(n_{\eta}-1\right) / 2}^{\left(n_{\eta}-1\right) / 2}\left|z_{2}\left(k_{\tau}-m_{\tau} \Delta_{\tau}, k_{\eta}-m_{\eta} \Delta_{\eta}\right)\right|^{2}}
$$

where $k_{\tau}$ and $k_{\eta}$ are the index of the range frequency and Doppler frequency samples, respectively, $\left(\Delta_{\tau}, \Delta_{\eta}\right)$ are the sensor sample spacing in the $\left(k_{\tau}, k_{\eta}\right)$ domain, and both $n_{\tau}$ and $n_{\eta}$ should be chosen to be odd integers. The stationarity and ergodicity assumptions are essential, and they impose a serious restriction upon the practical implementation of the proposed algorithm. As such, $Z_{1}$ and $Z_{2}$ should be slowly varying over the 2-D spectral window, therefore the size of the window should be small compared to the support region of the signals. Specifically, let $N_{\tau}$ and $N_{\eta}$ denote the total sample numbers in range-frequency and Doppler-frequency dimensions, respectively, then $n_{\tau} \ll N_{\tau}$ and $n_{\eta} \ll N_{\eta}$. Usually the window-size of $3 \times 3,5 \times 5$, or $5 \times 3$ is taken for practical implementation, and almost the same performance can be achieved. Table 1 summarizes the proposed algorithm.

Table 1. Summary of the proposed algorithm

\begin{tabular}{|c|c|}
\hline Problem & $\begin{array}{l}\text { Find } \beta \text { to solve the equation } \\
\text { where } \\
\qquad E\left[\left(Z_{1}-Z_{2, \text { cal }}\right) Z_{2}^{*}\right]=0 \\
\qquad Z_{2, \text { cal }}=\beta Z_{2} \\
\end{array}$ \\
\hline Optimum solution & $\beta=\frac{E\left[Z_{1} Z_{2}^{*}\right]}{E\left[\left|Z_{2}\right|^{2}\right]}$ \\
\hline Implementation form & $\begin{array}{l}\hat{\beta}\left(k_{\tau}, k_{\eta}\right)=\left.\frac{\sum_{m_{\tau}=-\left(n_{\tau}-1\right) / 2}^{\left(n_{\left.\tau^{-}-1\right) / 2}\right.} \Sigma_{m_{\eta}=-\left(n_{\eta}-1\right) / 2}^{\left(n_{\eta}-1\right) / 2} Z_{1}\left(k_{\tau}-m_{\tau} \Delta_{\tau}, k_{\eta}-m_{\eta} \Delta_{\eta}\right) Z_{2}^{*}\left(k_{\tau}-m_{\tau} \Delta_{\tau}, k_{\eta}-m_{\eta} \Delta_{\eta}\right)}{\left.\sum_{m_{\tau}=-(2) / 2}^{\left(n_{\tau}-1\right) / 2} \Sigma_{\left.m_{\eta}-1\right) / 2}^{\left(n_{\eta}-1\right)}\right|_{\eta}=-\left(n_{\eta}-1\right) / 2} Z_{2}\left(k_{\tau}-m_{\tau} \Delta_{\tau}, k_{\eta}-m_{\eta} \Delta_{\eta}\right)\right|^{2} \\
\text { both } n_{\tau} \text { and } n_{\eta} \text { should be chosen to be odd integers. }\end{array}$ \\
\hline
\end{tabular}

\section{Experiment with performance test}

In order to evaluate the performance of the proposed method, results concerning channel calibration are illustrated and investigated experimentally using real SAR data. For comparison, the results of the A2DC method are also illustrated. In the experiment, a $3 \times 3$ moving-window is used for the proposed method, which needs no iterative operation, and the iteration number of the A2DC method is 3, which is calculated according to the criteria presented in [9]. To begin with, a description of the experiment system is needed.

\subsection{Experiment system description}

An airborne SAR-GMTI experiment has been carried out and a set of three-channel X-band SAR data have been collected. The system and geometry parameters for the experiment system are listed in Table 2. The entire antenna is partitioned into three sub-apertures aligning along the track of the radar platform, and only the middle sub-aperture is used for transmission, whereas all the three parallel sub-apertures are used for reception simultaneously and coherently.

Table 2. System and geometry parameters

\begin{tabular}{|l|c|l|}
\hline Parameter & Symbol & Value \\
\hline Carrier frequency & $f_{c}$ & $9 \mathrm{GHz}$ \\
\hline Pulse repetition frequency & $P R F$ & $840 \mathrm{~Hz}$ \\
\hline Doppler bandwidth & $B_{\eta}$ & $530 \mathrm{~Hz}$ \\
\hline Doppler centroid frequency & $f_{D C}$ & $-90 \mathrm{~Hz}$ \\
\hline Platform velocity & $v_{p}$ & $106 \mathrm{~m} / \mathrm{s}$ \\
\hline Antenna separation & $d$ & $0.4 \mathrm{~m}$ \\
\hline
\end{tabular}


The channel calibration performance is evaluated using the real SAR data of the scene shown in Figure 1, whose resolution is approximately $8 \mathrm{~m} \times 8 \mathrm{~m}$. Particularly, we highlight the CNR versus Doppler frequency performance of the channels in Figure 2, which is created by estimating the CNR at each Doppler frequency bin in 2-D frequency domain. As can be observed, the CNR performance over Doppler frequency is modulated by the antenna beam pattern, and there is a considerable drop of the CNR in the sidelobe regions. Thus the signals in these sidelobe regions will be decorrelated due to the decrease of the CNR, whereas the ones within the mainbeam are highly correlated. This phenomenon will lend much insight to our analysis in the following discussion.



Figure 1. Selected scene for performance verification

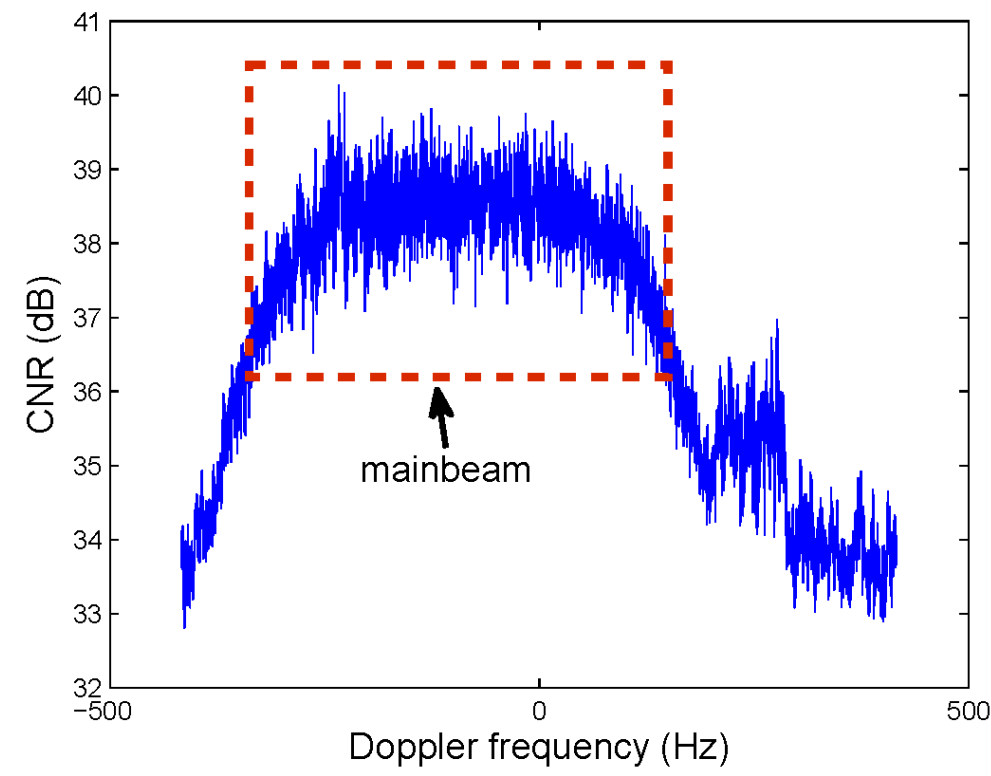

4.2. Testing results

Figure 2. CNR performance of the channels over Doppler frequency

The proposed implementation scheme in (18) is applied to the three channel data of the scene shown in Figure 1. In the processing, Channel 1 is selected as the reference channel, and the other two channels are adjusted to it. This calibration scheme is the same as the one presented in $[2,3,4]$. For the sake of clarity, only the processing results concerning Channels 1 and 2 are shown in the following. 
The channel imbalances before and after implementing the proposed method are shown in Figure 3 . It can be seen from Figs. $3 \mathrm{a}$ and $3 \mathrm{~b}$ that the original amplitude imbalance between the two channels is small, whereas the original phase imbalance is prominent. It can also be observed from Figs. 3c and $3 \mathrm{~d}$ that both amplitude and phase imbalances within the mainbeam are well compensated, whereas the imbalances within the sidelobe regions are badly compensated. This is because the signals within the mainbeam are highly correlated due to the high CNR, and the signals within the sidelobe regions are decorrelated by the noise due to the reduced CNR. It is a difficult task to calibrate the signal components if their correlation is low.



(a)

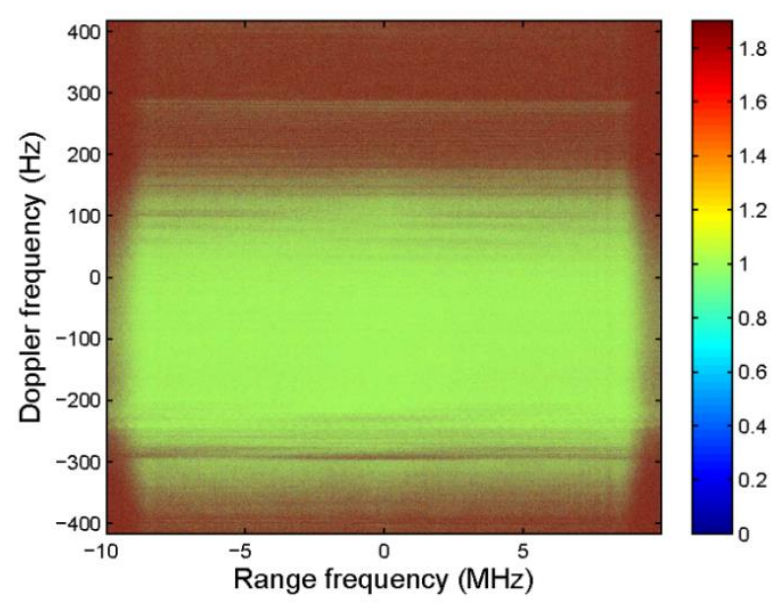

(c)

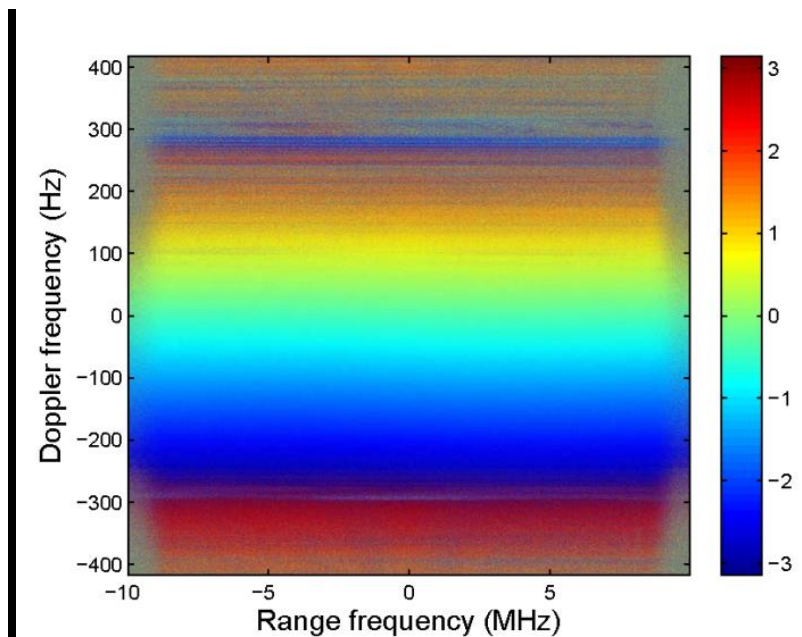

(b)

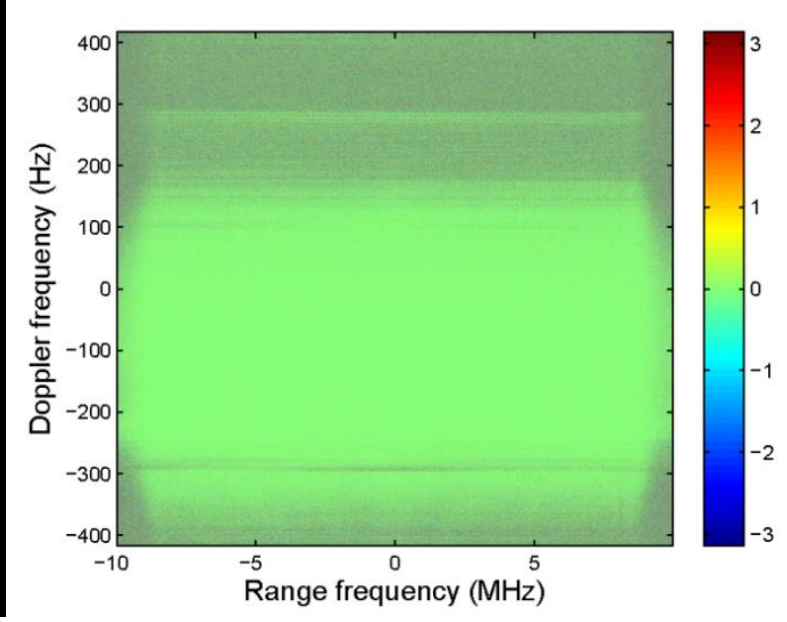

(d)

Figure 3. Channel imbalances between channel 1 and 2.(a) Original amplitude spectrum imbalance.(b) Original phase spectrum imbalance.(c) Residual amplitude imbalance after calibrating with the proposed method.(d) Residual phase imbalance after calibrating with the proposed method.

In Figure 4, a more dramatic indication of the channel calibration results is seen by creating the averaged amplitude spectrum plots and the angle histograms. Figs. $4 \mathrm{a}$ and $4 \mathrm{~b}$ are created by averaging the amplitude spectrum of the two channels along the range dimension. In Figure 4a, distinct original amplitude imbalances exist in both the mainbeam and sidelobe regions. In Figure $4 b$, the imbalances within the mainbeam are well compensated, whereas the ones within the sidelobe regions are enlarged. These enlarged imbalances could be explained by the random noises which are uncorrelated. Another possible explanation is the moving targets which are decorrelated by the target motion. Figs. $4 \mathrm{c}$ and $4 \mathrm{~d}$ show the angle histograms of the 2-D frequency domain interferometeric phase. As can be observed, the phases obtained before channel calibration are asymmetrically scattered in a wide range, whereas the ones obtained after channel calibration with the proposed method are symmetrically gathered around zero. Thus both the amplitude and phase imbalances are well compensated by the proposed method. 


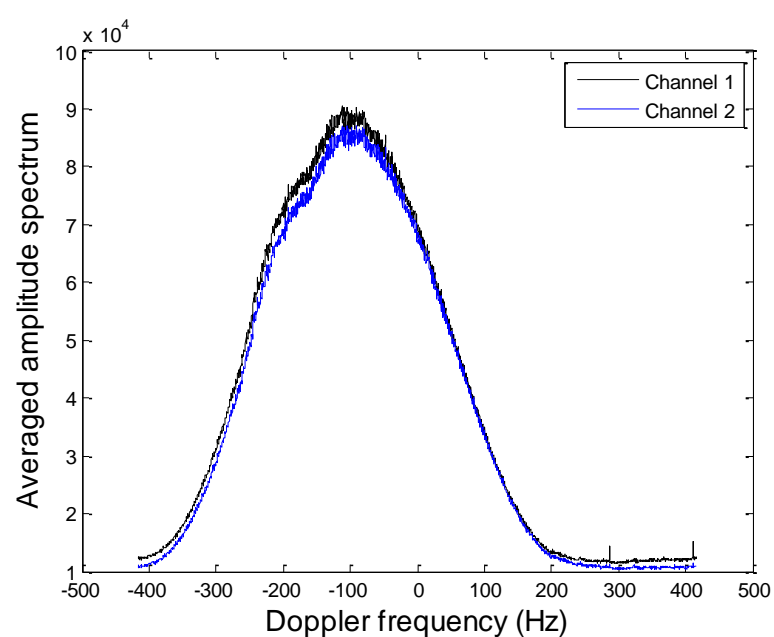

(a)

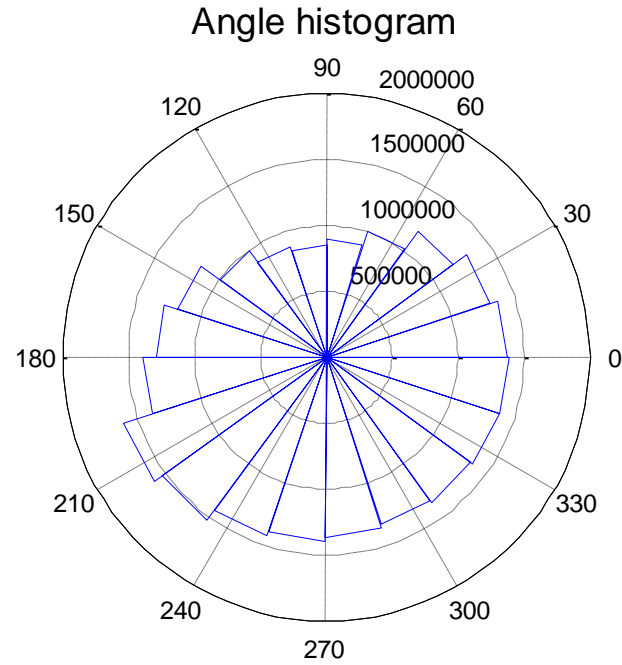

(c)

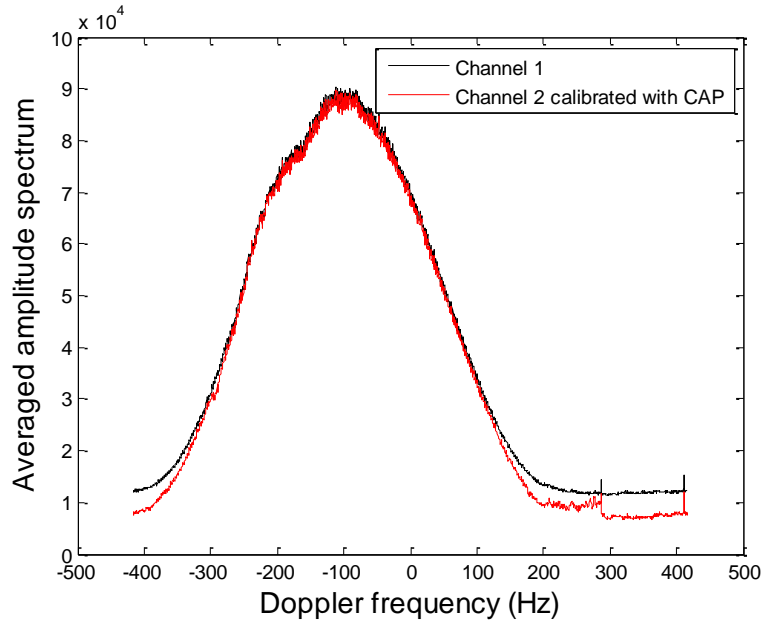

(b)

Angle histogram

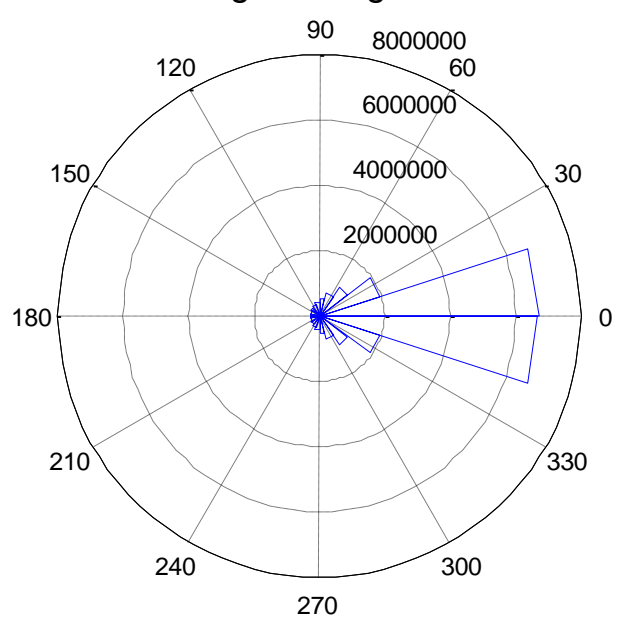

(d)

Figure 4. Amplitude and phase characteristics (a) Amplitude spectrum averaged along the range dimension before channel calibration.(b) Amplitude spectrum averaged along the range dimension after calibrating with the proposed method. (c) Angle histogram of the interferometric phase in 2D frequency domain before channel calibration.(d) Angle histogram of the interferometric phase in 2D frequency domain after calibrating with the proposed method.

Next, we compare the performance of the proposed method with the A2DC method. A convenient way to assess the performance is to examine the amplitude and phase imbalances between the two channels as shown in Figure 5. For comparison, four performance curves, i.e., the averaged amplitude spectra of the signals from channel 1, channel 2, channel 2 calibrated with the proposed method, and channel 2 calibrated with the A2DC method, are shown together in Figure 5a. The averaged amplitude ratio shown in Figure $5 \mathrm{~b}$ is created by dividing the averaged amplitude spectrum of channel 1 by the one of channel 2 (before or after channel calibration). For the sake of clarity, only the results within the mainbeam are shown in Figure 5b. We can observe that the performance of the proposed method is closer to the ideally calibrated case, in which the averaged amplitude ratio is 1 . Similar conclusion can be drawn from Figure 5c. As can be seen, the residual phase imbalance of the proposed method is closer to 0 rad, thus a better channel calibration performance of the proposed method can be concluded.

It is worth relating the results shown in Figure 5 with the CNR curve shown in Figure 2. As can be observed, both of the two methods behave well in high CNR conditions and badly when the CNR decreases, and there is a distinct improvement of the proposed method when the CNR is decreased. Thus, we conclude that the proposed method performs better in low CNR conditions. 


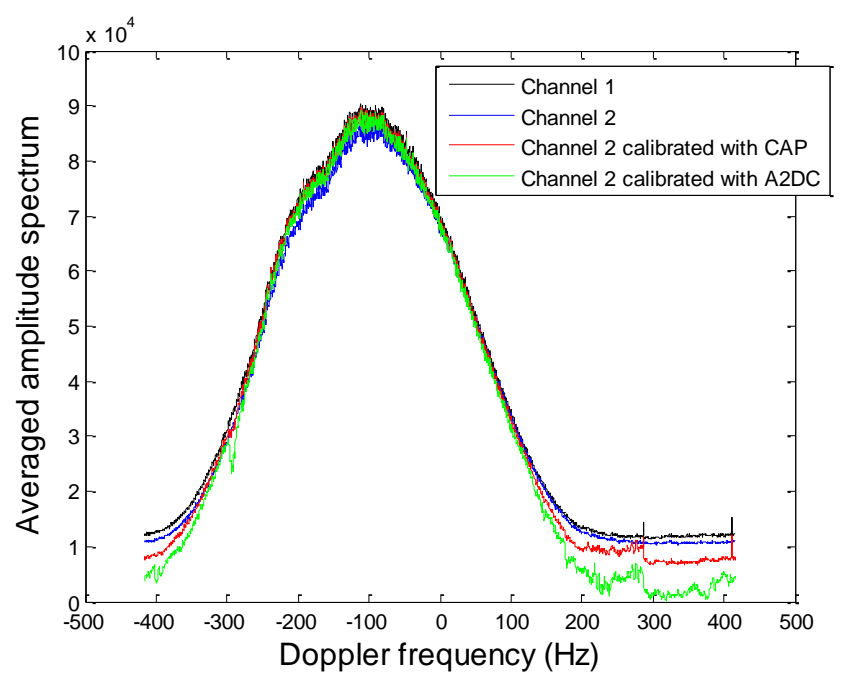

(a)

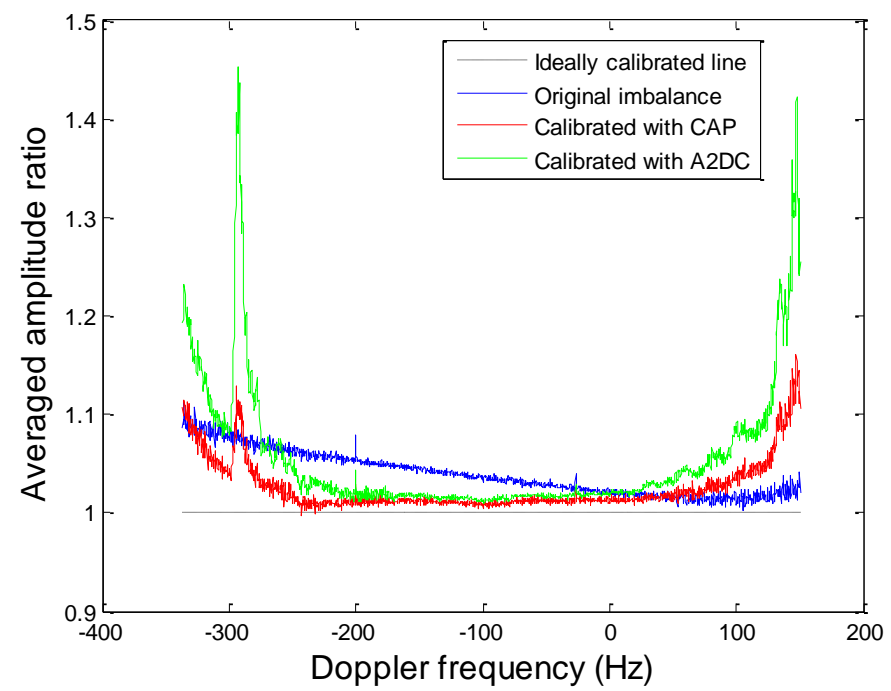

(b)

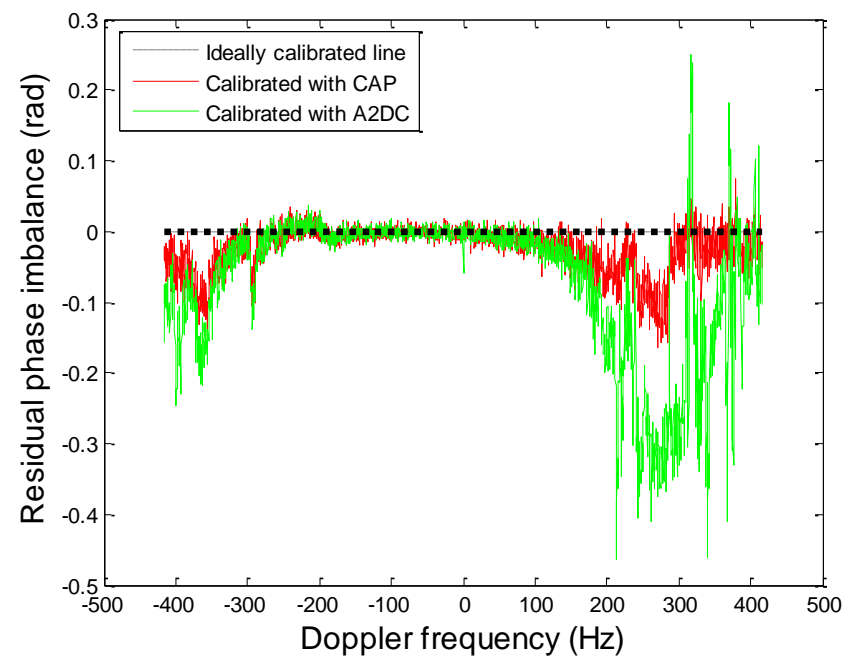

(c)

Figure 5. Amplitude and phase imbalances between channels 1 and 2. (a) Imbalances indicated by the averaged amplitude spectrum.(b) Averaged amplitude ratio of channel 1 to channel 2. Only the values within the mainbeam are shown for clarity.(c) Residual phase imbalances between channels 1 and 2. 
If the channels are well calibrated, the clutter suppression can be performed by simply subtracting pairs of the channels, i.e., by the so DPCA processing. Thus, the channel calibration performance is highly related to the clutter suppression performance, which in turn is closely related to the DOC as shown in (6). As such, both the DOC and the CSR can be employed as performance metrics to evaluate the channel calibration performance. Therefore, both the DOC and the CSR are computed from the real SAR data, and the results are shown in Table 3. For comparison, the results of both the proposed CAP method and the A2DC method are listed together. As can be seen, the two methods behave closely. Combined with the results shown in Figure 5, it should not be surprising that the proposed method sows better performance over A2DC.

Table 3. Quantitative comparative results
\begin{tabular}{|c|c|c|}
\hline Method & DOC & CSR $(\mathrm{dB})$ \\
\hline CAP & 0.9974 & 22.6693 \\
\hline A2DC & 0.9973 & 22.4983 \\
\hline
\end{tabular}

\section{Conclusions}

In this paper, we study a very general channel calibration problem, for which only the knowledge of the first two moments of the channel output signals is assumed, and no other distributional assumptions are made. Based on correlation analysis theory, an optimum solution to the general channel calibration problem is obtained. This optimum solution gives rise to the proposed channel calibration algorithm, which has the merits of needing no iterative operation and requiring a simple implementation form. Results with real SAR data show that the proposed algorithm is effective of calibrating the channels. It can be concluded that the proposed algorithm represents a generic solution to the general channel calibration problem, which both leads to a simple implementation form and requires the knowledge of just the first two moments.

\section{References}

1. Z.-Y. Chen and T. Wang, "Two-stage channel calibration technique for multichannel synthetic aperture radar-ground moving target indication systems," IET Radar, Sonar \& Navigation, vol. 8, no. 9, pp. 1116-1126, December 2014

2. Z.-Y. Chen, and T. Wang, "Unambiguous across-track velocity estimation of moving targets for multichannel SAR-GMTI systems," IET Signal Processing, December 2014

3. Z.-Y. Chen, T. Wang, and N. Ma, "Accurate baseline estimation for synthetic aperture radar-ground moving target indication systems based on co-registration and median filtering," IET Radar, Sonar \& Navigation, vol. 8, no. 6, pp. 607 - 615, July 2014

4. Z.-Y. Chen, T. Wang, and N. Ma, "Simple and robust baseline estimation method for multichannel SAR-GMTI systems," International Journal of Electronics, vol. 103, no. 7, pp. 1127-1137, July 2016

5. D. Cristallini, D. Pastina, F. Colone, and P. Lombardo, "Efficient Detection and Imaging of Moving Targets in SAR Images Based on Chirp Scaling," IEEE Transactions on Geoscience and Remote Sensing, vol. 51, no. 4, pp. 2403-2416, April 2013

6. J. H. G. Ender, "Space-time processing for multichannel synthetic aperture radar," IEE Electronics \& Communication Engineering Journal, vol. 11, no. 1, pp. 29-38, February 1999

7. J. H. G. Ender, "The airborne experimental multi-channel SAR system AER-II," in Proceedings of EUSAR, Königswinter, Germany, March 1996, pp. 49-52

8. M. R. Foster and N. J. Guinzy, "The coefficient of coherence: its estimation and use in geophysical data processing," Geophysics, vol. 32, no. 4, pp. 602-616, August 1967

9. C. H. Gierull, "Digital channel balancing of along-track interferometric SAR data," DRDC, Ottawa, ON, Canada, Technical Report TM 2003-024, March 2003

10. J. Guo, Z. F. Li, and Z. Bao, "Adaptive clutter suppression and resolving of velocity ambiguities for an experimental threechannel airborne SAR-GMTI system," IET Radar, Sonar \& Navigation, vol. 5, no. 4, pp. 426-435, 2011

11. S. M. Kay, "Fundamentals of statistical signal processing: estimation theory," Prentice Hall, New Jersey, USA, 1993

12. L. Lightstone, D. Faubert, and G. Rempel, "Multiple phase centre DPCA for airborne radar," in Proceedings of 1991 IEEE National Radar Conference, pp. 36-40, 1991

13. A. Moccia, and G. Rufino, "Spaceborne along-track SAR interferometry: Performance analysis and mission scenarios," IEEE Transactions on Aerospace and Electronic Systems, vol. 37, no. 1, pp. 199-213, January 2001

14. J. Qian, X. Lv, M. Xing, L. Li, and Z. Bao, "Motion parameter estimation of multiple ground fast-moving targets with a threechannel synthetic aperture radar," IET Radar, Sonar \& Navigation, vol. 5, no. 5, pp. 582-592, 2011

15. T. K. Sjogren, V. T. Vu, M. I. Pettersson, A. Gustavsson, and L. M. H. Ulander, "Moving Target Relative Speed Estimation and Refocusing in Synthetic Aperture Radar Images," IEEE Transactions on Aerospace and Electronic Systems, vol. 48, no. 3, pp. 2426-2436, July 2012

16. G. W. Stimson, Introduction to airborne radar, 2nd ed. New Jersey: SciTech Publishing, 1998

17. R. Touzi, A. Lopes, J. Bruniquel, and P. W. Vachon, "Coherence estimation for SAR imagery," IEEE Transactions on Geoscience and Remote Sensing, vol. 37, no. 1, pp. 135-149, January 1999

18. S. V. Vaseghi, “Advanced digital signal processing and noise reduction," 4th ed., John Wiley \& Sons, New York, USA, 2008

19. R. E. Walpole, R. H. Myers, S. L. Myers, and K. Ye, "Probability and statistics for engineers and scientists," 9th ed., Prentice Hall, New York, USA, 2012

20. T. Wang, Z. Bao, Z. Zhang, and J. Ding, "Improving coherence of complex image pairs obtained by along-track bistatic SARs using range-azimuth prefiltering," IEEE Transactions on Geoscience and Remote Sensing, vol. 46, no. 1, pp. 3-13, January 
2008

21. N. Wiener, "Generalized harmonic analysis," Acta Mathematica, vol. 55, no. 1, pp. 117-258, 1930

\section{Appendix}

In this appendix, two important properties concerning MMSE and BLUE of the proposed algorithm are highlighted. These properties can justify the optimum performance of the proposed algorithm.

\section{A.1 Optimum in the MMSE sense}

The key idea behind the MMSE criterion is that we want to find an optimum coefficient $\beta_{M M S E}$ such that, if the channel calibration is performed by (9), the resulting mean square error

$$
M S E=E\left[\left(Z_{1}-\beta_{M M S E} Z_{2}\right)^{2}\right]
$$

is minimum. We assume that the optimum coefficient is the one obtained from the proposed algorithm:

$$
\beta_{M M S E}=\beta_{C A P}
$$

where $\beta_{C A P}$ is the coefficient of the proposed method presented in (12). We proceed to confirm the validity of this assumption, and thus we shall show that the MSE is minimal. Let $Z_{2, c a l}^{\prime}=\beta^{\prime} Z_{2}$ be the signal calibrated with an arbitrary coefficient $\beta^{\prime}$, then the MSE is

$$
\begin{aligned}
E\left[\left(Z_{1}-Z_{2, c a l}^{\prime}\right)^{2}\right] & =E\left[\left(Z_{1}-\beta_{C A P} Z_{2}+\beta_{C A P} Z_{2}-\beta^{\prime} Z_{2}\right)^{2}\right] \\
& =E\left\{\left[\left(Z_{1}-\beta_{C A P} Z_{2}\right)+\left(\beta_{C A P}-\beta^{\prime}\right) Z_{2}\right]^{2}\right\} \\
& =E\left[\left(Z_{1}-\beta_{C A P} Z_{2}\right)^{2}\right]+\left(\beta_{C A P}-\beta^{\prime}\right)^{2} E\left[\left|Z_{2}\right|^{2}\right]+2\left(\beta_{C A P}-\beta^{\prime}\right) E\left[\left(Z_{1}-\beta_{C A P} Z_{2}\right) Z_{2}^{*}\right]
\end{aligned}
$$

Here, the last term equals to zero according to (11), and the second term is non-negative, thus it follows that, for any $\beta^{\prime}$,

$$
E\left[\left(Z_{1}-Z_{2, c a l}^{\prime}\right)^{2}\right] \geq E\left[\left(Z_{1}-\beta_{C A P} Z_{2}\right)^{2}\right]
$$

Hence, MSE is minimum if and only if $\beta^{\prime}=\beta_{C A P}$, thus the assumption in (20) is proofed.

From the above analysis, we can see that it is possible to design a channel calibration algorithm in the MMSE sense by implementing the correlation analysis processing.

\section{A.2 Optimum in the BLUE sense}

The BLUE of $\alpha$ shown in (7) can be determined with the knowledge of only the first two moments of the random signals, and this a priori knowledge is exactly the one required for the proposed algorithm. As such, we consider a fairly general statistical model, which is described as follows. Denote the data sets observed from the random signals $Z_{1}$ and $Z_{2}$ as $\left\{Z_{1}[1], Z_{1}[2], \cdots, Z_{1}[N]\right\}$ and $\left\{Z_{2}[1], Z_{2}[2], \cdots, Z_{2}[N]\right\}$, respectively. To derive the BLUE of $\alpha$, we restrict attention to the linear estimator of the form

$$
\hat{\alpha}=\sum_{n=1}^{N} d_{n}^{*} Z_{1}[n]
$$

where $d_{n}$ 's are constants yet to be determined. Among the class of linear estimator in (23), we further restrict attention to unbiased estimator, which is of the form

$$
E\{\hat{\alpha}\}=\sum_{n=1}^{N} E\left\{d_{n}^{*} Z_{1}[n]\right\}=\alpha
$$

Inserting (7) into (24), yields

$$
\alpha=\sum_{n=1}^{N} E\left\{d_{n}^{*} Z_{1}[n]\right\}=\sum_{n=1}^{N} E\left\{d_{n}^{*} Z_{2}[n]\right\} \alpha
$$


Thus the unbiased constraint yields the restriction

$$
\sum_{n=1}^{N} E\left\{d_{n}^{*} Z_{2}[n]\right\}=1
$$

Next, we examine the variance of the estimator:

$$
\operatorname{Var}\{\hat{\alpha}\}=\sum_{n=1}^{N} \operatorname{Var}\left\{d_{n}^{*} Z_{1}[n]\right\}=\sum_{n=1}^{N} E\left\{\left|d_{n}^{*} Z_{1}[n]\right|^{2}\right\}=\sigma_{1}^{2} \sum_{n=1}^{N} E\left\{\left|d_{n}\right|^{2}\right\}
$$

where $\sigma_{1}^{2}=E\left\{\left|Z_{1}\right|^{2}\right\}$

Now, we summarize the estimation problem. To find the BLUE is equivalent to find the linear estimator that is unbiased and has minimum variance. As such, according to the unbiased constraint in (26) and the variance in (27), we need to solve the equality-constrained optimization problem:

$$
\begin{array}{cl}
\operatorname{minimize} & \sigma_{1}^{2} \sum_{n=1}^{N} E\left\{\left|d_{n}\right|^{2}\right\} \\
\text { subject to } & \sum_{n=1}^{N} E\left\{d_{n}^{*} Z_{2}[n]\right\}=1 .
\end{array}
$$

The solution to this minimization problem is given by [11]:

$$
d_{n, o p t}=\frac{Z_{2}[n] / N}{E\left[\left|Z_{2}\right|^{2}\right]}
$$

Thus the BLUE is

$$
\hat{\alpha}_{B L U E}=\sum_{n=1}^{N} d_{n, o p t}^{*} Z_{1}[n]=\frac{E\left[Z_{1} Z_{2}^{*}\right]}{E\left[\left|Z_{2}\right|^{2}\right]}
$$

and this is exactly the coefficient obtained by the proposed algorithm shown in (12).

To summarize, the proposed algorithm can obtain an optimal estimate of the channel imbalances in the BLUE sense. 